European temperate forests (such as to tropical forests or to plant species other than trees). Moreover, if seed-dispersing animals are as crucial to the persistence of plants as this and other studies suggest $(28,29)$, then the combination of habitat loss with direct and indirect removal of animals, to which many of the world's most diverse forests are subject, is likely to have more drastic effects than either perturbation alone. In these circumstances, animaldispersed species might be more, not less, sensitive to habitat loss. This points to the maintenance of the network of plant-animal interactions as a comerstone of conservation policy and to the need for more studies of species responses to habitat loss.

\section{References and Notes}

1. R. V. Solé, J. Bascompte, Self Organization in Complex Ecosystems (Princeton Univ. Press, Princeton, N], 2007).

2. L. Fahrig, Biol. Conserv. 100, 65 (2001).

3. I. Hanski, Metapopulation Ecology (Oxford Univ. Press, Oxford, 1999).

4. M. Rees, R. Condit, M. Crawley, S. Pacala, S. Tilman, Science 293, 650 (2001).

5. E. I. Damschen, N. M. Haddad, J. L. Orrock, J. J. Tewksbury, D. J. Levey, Science 313, 1284 (2006).
6. B. C.-L. Chetkiewicz, C. C. St. Clair, M. S. Boyce, Annu. Rev. Ecol. Syst. 37, 317 (2006).

7. L. Conradt, T. J. Roper, C. D. Thomas, Oikos 95, 416 (2001).

8. G. Ferraz et al., Science 315, 238 (2007)

9. I. Hanski, M. Kuussaari, M. Nieminen, Ecology 75, 747 (1994).

10. Segundo Inventario Forestal Nacional (Ministerio de Agricultura, Pesca y Alimentación, Madrid, 1995).

11. Information on materials and methods is available as supporting material on Science Online.

12. F. González Manzaneque, Bosques Ibéricos: Una Interpretación Geobotánica (Planeta, Barcelona, 2005).

13. ]. Bascompte, M. A. Rodríguez, Ecol. Lett. 4, 417 (2001).

14. L. Fahrig, Annu. Rev. Ecol. Syst. 34, 487 (2003).

15. D. W. Purves, ]. Dushoff, J. Ecol. 93, 658 (2005).

16. I. Hanski, Nature 396, 41 (1998).

17. D. W. Purves, M. A. Zavala, K. Ogle, F. Prieto, J. M. Rey-Benayas, Ecol. Monogr. 77, 77 (2007).

18. K. Johst, R. Brandl, S. Eber, Oikos 98, 263 (2002).

19. ]. M. Gómez, Ecography 26, 573 (2003).

20. B. McEuen, L. M. Curran, Ecology 85, 507 (2004).

21. C. C. Smith, S. D. Fretwell, Am. Nat. 108, 499 (1974).

22. D. Sánchez-Gomez, F. Valladares, M. A. Zavala, New Phytol. 170, 795 (2006).

23. A. Purvis, P.-M. Agapow, J. L. Gittleman, G. M. Mace, Science 288, 328 (2000)

24. O. Spiegel, R. Nathan, Ecol. Lett. 10, 718 (2007).

25. P. Jordano, C. García, J. A. Godoy, ]. L. García-Castaño Proc. Natl. Acad. Sci. U.S.A. 104, 3278 (2007).
26. S. I. Higgins, R. Nathan, M. L. Cain, Ecology 84, 1945 (2003). 27. C. Restrepo, N. Gómez, S. Heredia, Ecology 80, 668 (1999).

28. J. M. Montoya, S. L. Pimm, R. V. Solé, Nature 442, 259 (2006).

29. J. Bascompte, P. Jordano, Annu. Rev. Ecol. Syst. 38, 567 (2007).

30. We thank the Ministerio de Medio Ambiente, Spain; R. Vallejo and ]. A. Villanueva for help with IFN2 data; S. Pacala for hospitality during data preparation; J. A. F. Diniz-Filho, L. M. Bini, and M. A. Olalla-Tárraga for help with phylogenetic analyses; and B. A. Hawkins, J. M. Montoya, D. Coomes, and ].-C. Svenning for comments on manuscripts. D.M. was supported by the Spanish Ministry for Education and Science (fellowship AP2004-0075). M.A.R. and M.A.Z. were supported by the Spanish Ministry for Education and Science (grants CGL2006-03000/BOS and CGL2005-05830-C03-01/BOS, respectively).

Supporting Online Material

www.sciencemag.org/cgi/content/full/1158404/DC1

Materials and Methods

Figs. S1 to S3

Tables. S1 to S4

References

28 March 2008; accepted 15 May 2008

Published online 5 June 2008;

10.1126/science. 1158404

Include this information when citing this paper.

\title{
VelB/VeA/LaeA Complex Coordinates Light Signal with Fungal Development and Secondary Metabolism
}

\section{Özgür Bayram, ${ }^{1}$ Sven Krappmann, ${ }^{1 *}$ Min Ni, ${ }^{2}$ ]in Woo Bok, ${ }^{3}$ Kerstin Helmstaedt, ${ }^{1,4}$ Oliver Valerius, ${ }^{1}$ Susanna Braus-Stromeyer, ${ }^{1}$ Nak-Jung Kwon, ${ }^{2}$ Nancy P. Keller, ${ }^{3}$ Jae-Hyuk Yu, ${ }^{2}$ Gerhard H. Braus ${ }^{1} \dagger$}

Differentiation and secondary metabolism are correlated processes in fungi that respond to light. In Aspergillus nidulans, light inhibits sexual reproduction as well as secondary metabolism. We identified the heterotrimeric velvet complex VelB/VeA/LaeA connecting light-responding developmental regulation and control of secondary metabolism. VeA, which is primarily expressed in the dark, physically interacts with VelB, which is expressed during sexual development. VeA bridges VelB to the nuclear master regulator of secondary metabolism, LaeA. Deletion of either velB or veA results in defects in both sexual fruiting-body formation and the production of secondary metabolites.

$\mathrm{S}$ econdary metabolites of fungi include "friends and foes" of human health, such as Aspergillis' production of penicillin (1) and the carcinogenic aflatoxin precursor sterigmatocystin (ST), respectively (2). Secondary metabolic pathways are often tightly correlated with the fungal developmental program and response to external cues including light. The mold Asper-

${ }^{1}$ Institute of Microbiology and Genetics, Georg August University, D-37077 Göttingen, Germany. ${ }^{2}$ Department of Bacteriology and Department of Genetics, University of Wisconsin-Madison, Madison, WI 53706, USA. ${ }^{3}$ Department of Plant Pathology and Medical Microbiology and Immunology, University of WisconsinMadison, Madison, WI 53706, USA. ${ }^{4}$ Deutsche Forschungsgemeinschaft (DFG) Research Center for Molecular Physiology of the Brain (CMPB), D-37077 Göttingen, Germany.

*Present address: Zentrum für Infektionsforschung, University of Würzburg, D-97070 Würzburg, Germany. †To whom correspondence should be addressed. E-mail: gbraus@gwdg.de gillus nidulans forms airborne asexual spores in light but preferentially undergoes sexual reproduction in the dark $(3,4)$. The latter results in the

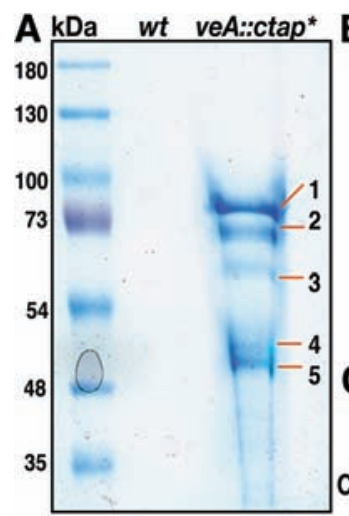

formation of sexual fruit bodies called cleistothecia, which consist of different cell types, and an increase in secondary metabolism (5). Mutations resulting in defects in fungal development often also impair secondary metabolism (6). There is genetic evidence for a connection between fruitbody formation, secondary metabolism, and light in A. nidulans, but the molecular mechanism is not known (7-9). One candidate for such a bridge is the conserved velvet protein encoded by the $v e A$ gene (10-12), whose expression increases during sexual development (7). VeA transport into the nucleus is inhibited by light (13). It acts as a negative regulator of asexual development (14) and antibiotic biosynthesis (15).

Biosynthetic genes for fungal secondary metabolite are often clustered and regulated by pathway-specific transcription factors $(16,17)$. Secondary metabolism is also regulated at an upper hierarchic level by a global epigenetic control mechanism. The nuclear LaeA protein is present in numerous fungi and is a master regulator of secondary metabolism in Aspergilli (18).

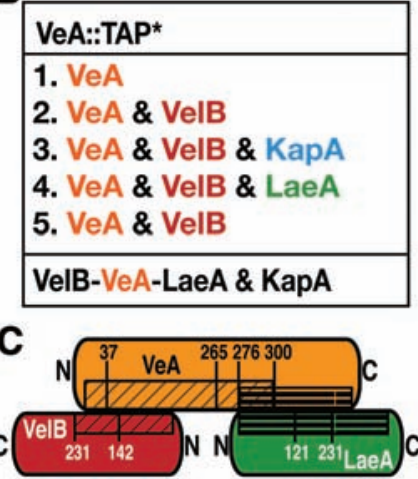

Fig. 1. Identification of $\mathrm{VeA}$ associated proteins in $A$. nidulans. (A) Brilliant blue G-stained 10\% SDS-polyacrylamide gel electrophoresis of TAP procedure for VeA. $k D$, kilodaltons. (B) The polypeptides identified from the bands of affinity purification belong to corresponding proteins (details in table S4). (C) Domain mapping of the interactions based on $\mathrm{Y} 2 \mathrm{H}$ data (fig. S2). N, N terminus; $\mathrm{C}, \mathrm{C}$ terminus. 
The deletion of laeA, although not reported to affect morphological and developmental processes, results in silencing of numerous secondary metabolite gene clusters, including those responsible for the syntheses of the antibiotic penicillin as
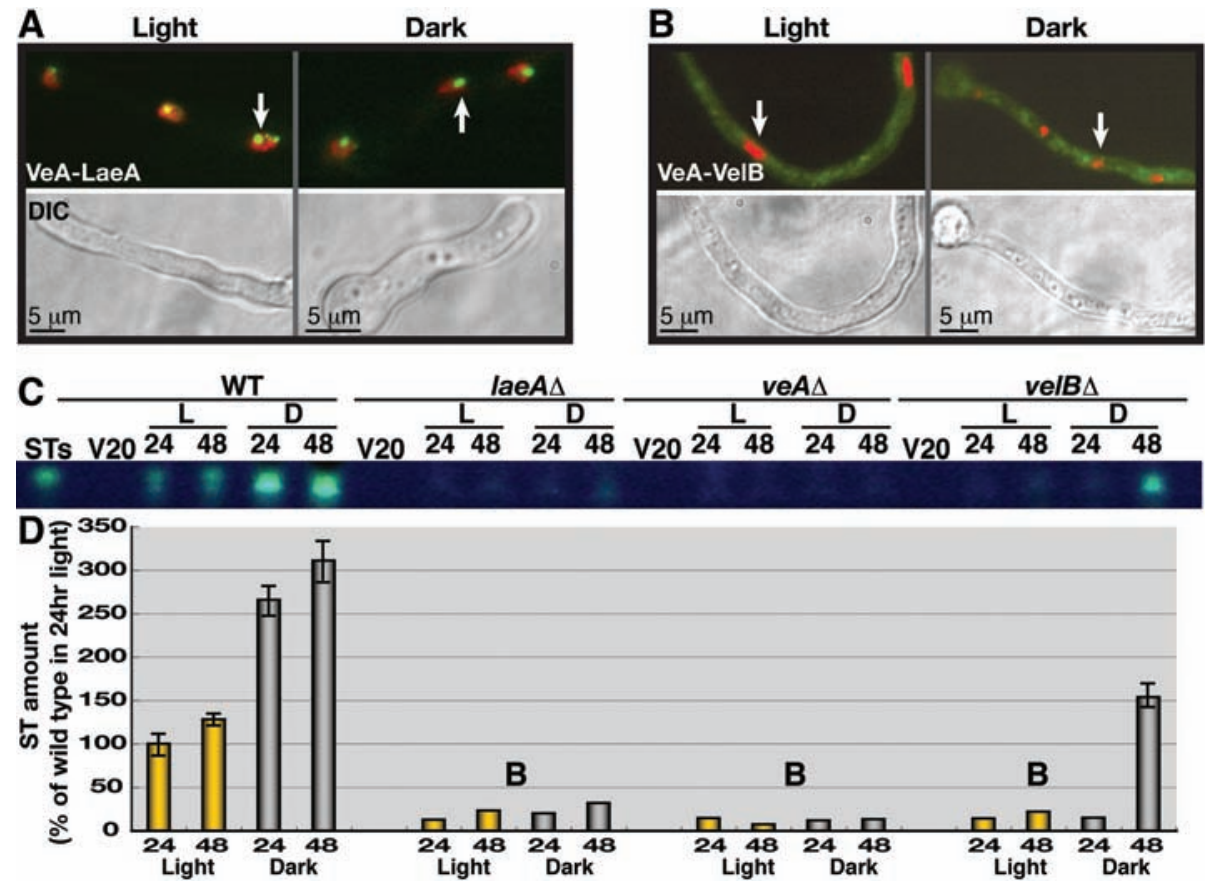

Fig. 2. BiFC studies of velvet complex components and their effect on ST production. (A) Enhanced yellow fluorescent protein fused to the $\mathrm{N}$ terminus of veA gene (N-EYFP::VeA) interacts with C-EYFP::LaeA in vivo, which is indicated as yellowish green specks in the nucleus. [Histone $2 \mathrm{~A}$ red fluorescent protein (H2A::mRFP) fusion visualizes the entire nucleus.] Interaction does not take place in the whole nucleus but in certain points (gene clusters) that LaeA probably acts on (indicated by arrows). Differential interference contrast (DIC) shows hyphal cells. (B) N-EYFP::VeA fusion protein interacts with C-EYFP::VelB in the cytoplasm and nucleus. (C) ST production in respective mutant backgrounds and WT at different time points. STs, ST standard; V20, 20 hours vegetative growth; L, light; D, dark. 24 and 48 hour time points are shown. (D) Quantification of ST production using thin layer chromatography: In the dark, more ST is produced in the WT. Deletion of either laeA or veA results in no ST above background (denoted by B) fluctuations. Loss of velB results in basal ST production in dark.
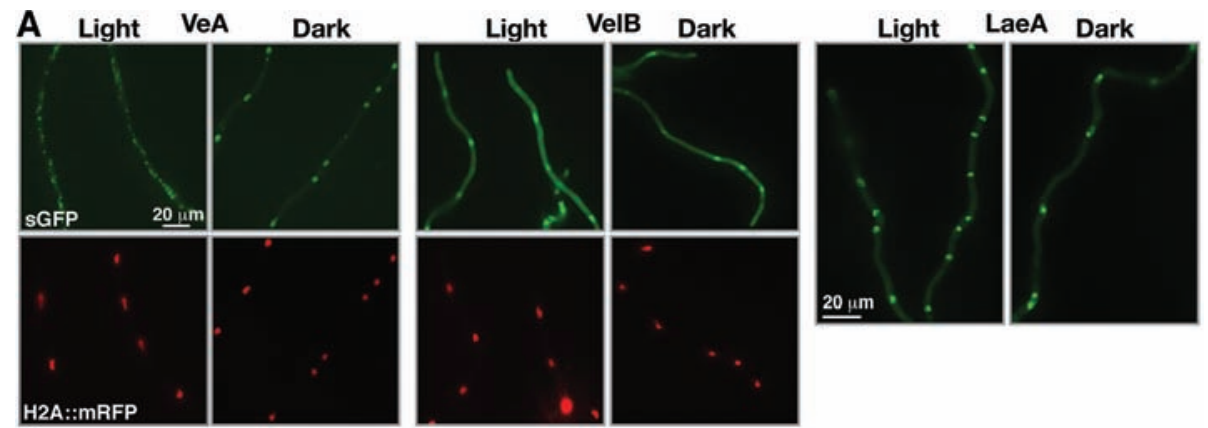

B
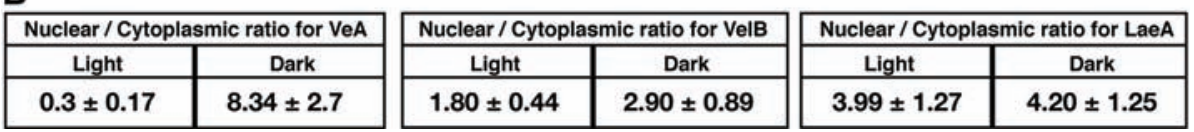

Fig. 3. Subcellular localization of the subunits of the velvet complex. (A) VeA-, LaeA-, and VelB-sGFP localizations in the presence or absence of light. VeA-sGFP shows light-dependent nuclear enrichment (counterstained with H2A::mRFP for visualization of the entire nucleus). (B) Nuclear/cytoplasmic GFP signal ratio of 100 hyphal cells each (Openlab software 5.0.1) (28). Growth in the dark results in increased nuclear and decreased cytoplasmic fluorescence for VeA. VelB and LaeA distribution is hardly affected by illumination.
(19) prevents heterochromatin maintenance of some clusters (20).

We used tandem affinity purification (TAP) (21-23) to identify VeA-interacting proteins (Fig. $1 \mathrm{~A}$ and fig. S1A) (24). Final eluates of dark- and light-grown $A$. nidulans carrying the functional $v e A$ gene tagged at its $\mathrm{C}$ terminus by TAP tag (veA::ctap ${ }^{*}$ ) were analyzed by mass spectrometry. We identified the velvet-like protein $\mathrm{B}$ (VelB) (fig. S3, A and B), the regulator LaeA, and the $\alpha$ importin KapA as proteins that interact with VeA in the dark (Fig. 1B and table S4). In the light, tagged VeA is hardly expressed (fig. S1B) and only copurifies with VelB. Reciprocal affinity purifications of tagged VelB and LaeA in the dark confirmed the interaction partners, except for the $\alpha$ importin KapA (fig. S1, C and D). Only tagged VelB can additionally recruit the regulator of sporogenesis VosA in the dark (25), which seems to be an alternative binding partner for this protein.

Yeast two-hybrid (Y2H) analysis (26) confirmed the VeA-VelB and VeA-LaeA interactions, where VelB and LaeA do not interact in this assay, suggesting that VeA acts as a bridge between VelB and LaeA (Fig. 1C). The Y2H VosA-LaeA interaction supports a role of LaeA in development (fig. S2). The C-terminal part of VeA interacts with LaeA, whereas the $\mathrm{N}$-terminal part of VeA, which includes the nuclear localization signal (NLS), is required for interaction with VelB (Fig. 1C and fig. S2). VelB, which is conserved in the fungal kingdom, shares $18 \%$ amino acid identity with VeA but has no typical NLS (fig. S3B). Transcript analysis reveals that velB expression increases like that of veA (7) at late developmental stages (fig. S3C). The VeA-LaeA and VeA-VelB interactions were visualized by bimolecular fluorescence complementation (BiFC) in living cells (27). Distinct fluorescent specks show that the VeA-LaeA interaction occurs in the nucleus, whereas VeA and VelB interact in the cytoplasm and within the nucleus (Fig. 2, A and B).

The physical interaction of VeA with VelB, as well as with LaeA, leads to the prediction that $\mathrm{VeA}$ and VelB are functionally interdependent. Similar to $v e A \Delta$, the velB $\Delta$ mutant (fig. S5A) no longer displays a light-dependent developmental pattern and is unable to form sexual fruit bodies, even in the dark. Asexual sporulation in velB $\Delta$ is impaired but not as strongly as in a $v e A$ deletion strain. Reintroduction of the $v e l B$ locus fully rescued all of the defects (fig. S5A). The veA $\Delta$ velB $\Delta$ double mutant exhibited a near-identical phenotype to that of the veAs single mutant. Neither $v e l B$ overexpression in a $v e A \Delta$ background nor $v e A$ overexpression in a velB $\Delta$ background rescued the defects of the individual mutants; likewise, laeA overexpression could not rescue secondary metabolite defects of veA $\Delta$ (fig. S6). Unlike overproduction of VeA, overexpression of $v e l B$ in a $v e A+$ background does not cause excessive production of cleistothecia, but it induces a twofold increase in asexual sporulation in comparison to the wild type (WT). This suggests that VeA 


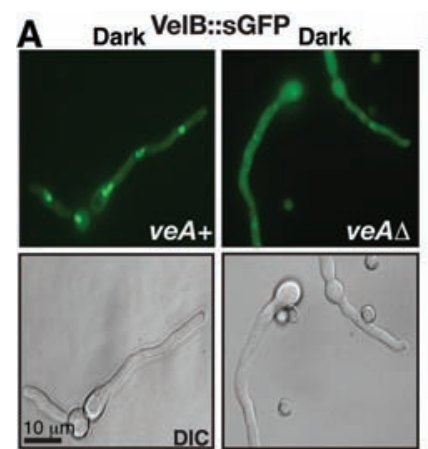

B

\begin{tabular}{|c|c|}
\hline \multicolumn{2}{|c|}{ Nuclear / Cytoplasmic ratio for VelB } \\
\hline in veAt+ & in veA $\Delta$ \\
\hline $2.90 \pm 0.89$ & $1.04 \pm 0.29$ \\
\hline
\end{tabular}

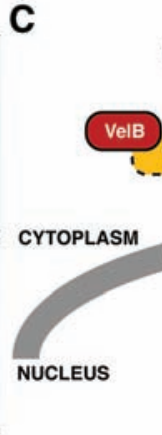
Less secondary metabolism
(ST production)

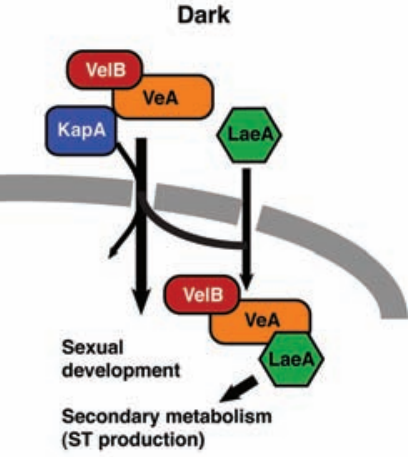

Fig. 4. VeA supports nuclear localization of VelB and formation of the velvet complex. (A) Fluorescence patterns in strains expressing velB:::sgfp in the dark in veA+ and veA $\Delta$ backgrounds. (B) Nuclear/ cytoplasmic GFP signal ratio of 100 hyphal cells each. Nuclear signal intensity is higher in the veA+ strain background than in veA $\Delta$. (C) Model: (Light) VeA is mostly retained in the cytoplasm, VelB supports asexual spore formation, and LaeA shows low activity. (Dark) An increased amount of VeA is imported into the nucleus by KapA and, in addition, supports the nuclear transport of VelB. Dotted lines indicate the decreased amount of VeA that is present in the cell in the light and the impairment of VeA nuclear transport in the light. VelB/NeA control development and LaeA activity by formation of the velvet complex that affects secondary metabolite clusters expression.

controls the number of sexual structures, whereas VelB has additional developmental functions.

Secondary metabolism is impaired in veA $\Delta$, resulting in a similar brownish pigment $(8)$ as is produced by the velBA strain. Changes in gene expression and in LaeA activity were monitored in the veA $A$ and velB $\Delta$ strains (Fig. 2, C and D, and figs. S5, B and $\mathrm{C}$, and $\mathrm{S} 7$ ). ST production is abolished in $v e A \Delta$ and lae $A \Delta$ strains. In contrast, reduced and delayed but significant ST production in velB $\Delta$ suggests residual activity of a VeA/LaeA complex in the dark

$\mathrm{VeA}$ is enriched in the nucleus in the dark (13), whereas VelB was found in both the nucleus and the cytoplasm and is hardly affected by illumination (Fig. 3, A and B). Because LaeA is constitutively nuclear (Fig. 3, A and B) (18) and the interaction of VeA and LaeA occurs in the nucleus (Fig. 2A), VelB has to enter the nucleus, despite the lack of an obvious NLS to fully control LaeA. Localization of the VelB-sGFP fusion protein (where GFP is green fluorescent protein) in a $v e A \Delta$ background is shifted toward the cytoplasm, whereas the presence of VeA increases the nuclear localization of VelB (Fig. 4, $A$ and $B)$. This suggests that VeA can assist VelB to allow an enhanced transport into the nucleus.

Our data suggest that the mechanism underlying the coordinated regulation of sexual development and secondary metabolism in A. nidulans might be the interaction between the key developmental regulatory complex VelB/VeA and LaeA. We propose that in the dark the VelB/VeA/LaeA velvet complex interaction controls and presumably supports the epigenetic activity of LaeA, which subsequently controls the expression of secondary metabolite gene clusters. In the light, this interaction is diminished because we find less VeA protein, and the entrance of the bridging factor VeA to the nucleus is decreased. Because the absence of LaeA has a minor impact on development, VeA and VelB have presumably additional functions in fungal differentiation. This is also supported by the identification of VosA, a recently identified regulator of fungal sporogenesis (25), as an additional binding partner of VelB (fig. S1, C and D, and table S4).

A. nidulans produces many compounds relevant to biotechnology and human health and is a well-suited model for the analysis of the interplay between secondary metabolism, light, and differentiation. $A$. nidulans grows vegetatively in the soil by hyphal tip extension until competent for development and secondary metabolism (3). Light triggers asexual development, corresponding to the release of high numbers of asexual spores (conidia) into the environment. These phenotypes correlate with the light-dependent cytoplasmic localization of VeA, the constitutive nuclear function of LaeA, and the partial nuclear localization of VelB, respectively. Under light conditions, when low amounts of VeA and VelB are present in the nucleus, the secondary metabolism regulator LaeA seems to be primarily active in those hyphae that are not exposed to light. Accordingly, the deletion of laeA results in a loss of mycelial pigmentation at the bottom of the colony (18).

The newly described fungal protein VelB, in conjunction with VeA, connects light-dependent development to LaeA-controlled secondary metabolism in A. nidulans. We present evidence that the formation of this complex is the molecular basis that synchronizes developmental and metabolic changes to the disappearance of light. We propose to designate this trimeric complex the velvet complex. We suggest that $\mathrm{VelB} / \mathrm{VeA}$ is part of the epigenetic control of chromatin remodeling by modulating LaeA methyltransferase activity
(16-18). We propose a scenario (Fig. 4C) in which VeA is functionally active in the dark, forms a complex with increased amounts of VelB, and enhances the transport of VelB to the nucleus. Because VeA and VelB are both partially nuclear, even in the light, we presume a certain threshold is probably necessary to initiate sexual development and control LaeA.

Fungal morphogenesis and secondary metabolism have traditionally been viewed as separate fields. Our studies on the VelB/VeA/LaeA velvet complex elucidate the molecular mechanisms underlying the intimate relation between fungal development and secondary metabolism.

\section{References and Notes}

1. A. A. Brakhage, FEMS Microbiol. Lett. 148, 1 (1997).

2. J. Hicks, K. Shimizu, N. P. Keller, in The Mycota, vol. 11, F. Kempken, Ed. (Springer, Berlin, 2002), pp. 55-69.

3. T. H. Adams, ]. K. Wieser, ].-H. Yu, Microbiol. Mol. Biol. Rev. 62, 35 (1998).

4. J. Purschwitz et al., Curr. Biol. 18, 255 (2008).

5. G. H. Braus, S. Krappmann, S. Eckert, in Molecular Biology of Fungal Development, H. D. Osiewacz, Ed. (Marcel Dekker, New York, 2002), pp. 215-244.

6. A. M. Calvo, R. A. Wilson, J. W. Bok, N. P. Keller, Microbiol. Mol. Biol. Rev. 66, 447 (2002).

7. H. Kim et al., Fungal Genet. Biol. 37, 72 (2002).

8. N. Kato, W. Brooks, A. M. Calvo, Eukaryot. Cell 2, 1178 (2003).

9. S. Busch, S. E. Eckert, S. Krappmann, G. H. Braus, Mol. Microbiol. 49, 717 (2003)

10. S. Krappmann, Ö. Bayram, G. H. Braus, Eukaryot. Cell 4, 1298 (2005).

11. S. Li et al., Mol. Microbiol. 62, 1418 (2006).

12. ]. Dreyer, H. Eichhorn, E. Friedlin, H. Kürnsteiner, U. Kück, Appl. Environ. Microbiol. 73, 3412 (2007).

13. S. M. Stinnett, E. A. Espeso, L. Cobeño, L. Araújo-Bazán, A. M. Calvo, Mol. Microbiol. 63, 242 (2007).

14. J. L. Mooney, L. N. Yager, Genes Dev. 4, 1473 (1990).

15. P. Spröte, A. A. Brakhage, Arch. Microbiol. 188, 69 (2007).

16. C. P. Woloshuk et al., Appl. Environ. Microbiol. 60, 2408 (1994).

17. N. P. Keller, G. Turner, ]. W. Bennett, Nat. Rev. Microbiol. 3, 937 (2005)

18. J. W. Bok, N. P. Keller, Eukaryot. Cell 3, 527 (2004).

19. J. W. Bok, D. Noordermeer, S. P. Kale, N. P. Keller, Mol. Microbiol. 61, 1636 (2006).

20. E. K. Shwab et al., Eukaryot. Cell 6, 1656 (2007).

21. O. Puig et al., Methods 24, 218 (2001).

22. J. S. Rohila, M. Chen, R. Cerny, M. E. Fromm, Plant J. 38 172 (2004).

23. S. Busch et al., Proc. Natl. Acad. Sci. U.S.A. 104, 8089 (2007).

24. Materials and methods are available as supporting material on Science Online.

25. M. Ni, J. H. Yu, PLoS One 2, e970 (2007).

26. J. Gyuris, E. Golemis, H. Chertkov, R. Brent, Cell 75, 791 (1993).

27. C. D. Hu, Y. Chinenov, T. K. Kerppola, Mol. Cell 9, 789 (2002).

28. Openlab software 5.0.1, www.improvision.com/.

29. We thank V. Pretz, G. Heinrich, V. Große, and Ö. S. Bayram for technical assistance. This project was supported by the DFG, CMPB, Volkswagen Vorab, and Fonds der Chemischen Industrie to G.H.B; Hatch (WIS04667) and NSF (MCB-0421863) grants to ].-H.Y.; and an NSF grant (MCB-0236393) to N.P.K. The GenelD for velB is 2876142.

\section{Supporting Online Material}

www.sciencemag.org/cgi/content/full/320/5882/1504/DC1

Materials and Methods

Figs. $\mathrm{S} 1$ to $\mathrm{S7}$

Tables S1 to S4

References

30 January 2008; accepted 29 April 2008

$10.1126 /$ science. 1155888 